\title{
Avaliação do desempenho e comportamento ingestivo de ovinos recebendo ração extrusada em diferentes relações volumoso e concentrado
}

\author{
Gilberto de Lima Macedo Junior ${ }^{1 *}$, Paulo Arthur Cardoso Ruela ${ }^{1}$ \\ Karla Alves Oliveira ${ }^{1}$, Carolina Moreira Araújo ${ }^{1}$, \\ Laura Ferrari Monteiro Varanis ${ }^{1}$, Tamires Soares de Assis ${ }^{1}$
}

\begin{abstract}
RESUMO
Objetivou-se avaliar o efeito da utilização de ração extrusada em diferentes relações volumoso:concentrado (V:C) sobre o comportamento ingestivo, medidas biométricas e ganho de peso de ovinos de classes sexuais distintas. $\mathrm{O}$ experimento foi realizado na Fazenda Experimental Capim Branco, pertencente à Universidade Federal de Uberlândia, nos meses de julho a setembro de 2015, sendo 15 dias destinados à adaptação dos animais e 90 dias para coleta de dados. Foram utilizados 20 ovinos cruzados $1 / 2$ Dorper e $1 / 2$ Santa Inês, sendo oito machos e doze fêmeas, com idade de aproximadamente quatro meses e peso corporal médio de $34 \mathrm{~kg}$, distribuídos aleatoriamente em quatro baias de piso ripado. Os tratamentos consistiram de diferentes relações V:C extrusado: 70C:30V, $60 \mathrm{C}: 40 \mathrm{~V}, 50 \mathrm{C}: 50 \mathrm{~V}, 40 \mathrm{C}: 60 \mathrm{~V}$. A cada 15 dias foram mensurados as medidas biométricas e o ganho de peso dos animais, com auxílio de fita métrica, régua e balança apropriadas. $\mathrm{O}$ comportamento ingestivo foi realizado no início e final do período experimental. $\mathrm{O}$ delineamento experimental utilizado foi inteiramente ao acaso em arranjo fatorial (sexo $\mathrm{x}$ níveis) com parcelas subdivididas. As variáveis foram comparadas por equação de regressão a $5 \%$ de probabilidade. As médias referentes a sexo foram comparadas pelo teste SNK a $5 \%$ de probabilidade. Não houve efeito das diferentes relações $\mathrm{V}: \mathrm{C}$ sobre o escore de condição corporal (ECC) e nem sobre os parâmetros biométricos. O ECC foi influenciado pelos períodos de avaliação e pelo sexo dos animais. Observaram-se diferenças significativas das distintas classes sexuais sobre o ganho médio diário, com superioridade dos machos. Houve interação entre sexo e período para ócio, tratamento e período para ruminação e interação entre tratamento e período para alimentação, sendo este último maior nos períodos iniciais. A ração extrusada provoca alterações na condição corporal dos ovinos, afetando positivamente o ganho de peso e alterando o comportamento ingestivo.
\end{abstract}

Palavras-chave: Biometria, Extrusão, Ganho de peso, Ovis Aries

\footnotetext{
${ }^{1}$ Faculdade de Medicina Veterinária da Universidade Federal de Uberlândia

* Corresponding author: gilberto.macedo@ufu.br Universidade Federal de Uberlândia, Faculdade de Medicina Veterinária. (34) 25126816
} 


\section{Introdução}

A extrusão é um processo que combina calor, umidade e força mecânica para modificar as características da ração. Através desse processamento é possível melhorar o valor nutritivo dos alimentos volumosos e concentrados, sendo capaz de reduzir fatores antinutricionais. Além disso, no processo de extrusão tem-se como resultado uma expansão da mistura de ingredientes, promovendo assim uma maior gelatinização do amido e aumentando a exposição de nutrientes contidos no interior das células vegetais (LIMA JÚNIOR et al., 2011), consequentemente melhorando a utilização do amido e diminuindo a taxa de degradação da proteína dentro do rúmen. As rações extrusadas têm sido amplamente utilizadas com o objetivo de melhorar o desempenho dos animais e facilitar o manejo alimentar nos diferentes sistemas de produção.

São poucos os trabalhos que avaliam o efeito de alimentos extrusados sobre diferentes parâmetros produtivos (WERNERSBACH FILHO, 2003; AMARAL et al., 2007; LIMA JÚNIOR et al., 2011) havendo assim uma lacuna a ser preenchida sobre impactos destes alimentos em medidas de desempenho animal. O peso é um dos primeiros e mais importante índice resgistrado, permitindo conhecer e avaliar a capacidade produtiva dos animais e seu desenvolvimento. A determinação do peso corporal prediz o desempenho na produção de carne, atua sobre capacidade de reprodução e indica o melhor momento de levar o animal ao abate.

Além do peso, as avaliações de medidas biométricas nos animais de interesse zootécnico são muito empregadas para conhecer o desenvolvimento das diferentes partes que compõem o exterior dos animais e com isso avaliar o desempenho animal. Os efeitos do manejo nutricional podem ser facilmente identificados nas diversas mensurações biométricas e nas análises de comportamento ingestivo. As avaliações de condição corporal juntamente com as demais medidas biométricas têm alta correlação com a 
qualidade de carcaça de cordeiros para abate e, nas fêmeas está intimamente ligado à produção de cordeiros (KANITZ et al., 2014), impactando significativamente o índice produtivo e reprodutivo do rebanho.

Em conjunto com as mensurações supracitadas, análises de consumo e comportamento ingestivo são necessárias para estabelecimento da correlação com desempenho dos animais, identificação e correção de possíveis falhas no manejo alimentar e nutricional. Conjuntamente ao conhecimento do comportamento ingestivo, as mensurações de consumo são de extrema importância em estudos de nutrição de ruminantes, pois dele vai depender a quantidade total de nutrientes que o animal recebe para produção, saúde e reprodução (COELHO DA SILVA, 2006). Frente à diversidade de fatores que influenciam o padrão de comportamento ingestivo dos animais, é necessário compreender como os alimentos extrusados provocam diferentes respostas sobre os parâmetros zootécnicos e as suas consequências para a economia do sistema de produção.

Nesse sentido, objetivou-se com esse estudo avaliar o efeito da utilização de ração extrusada em diferentes relações volumoso:concentrado (V:C) sobre o comportamento ingestivo, escore de condição corporal, medidas biométricas e ganho de peso de ovinos de classes sexuais distintas.

\section{Material e métodos}

O experimento foi realizado na Universidade Federal de Uberlândia no período de julho a setembro de 2015 , sendo 15 dias para adaptação e 90 dias para as coletas. Foram utilizados 20 ovinos cruzados $1 / 2$ Dorper e 1/2 Santa Inês, sendo oito machos e doze fêmeas, com idade de aproximadamente quatro meses e com peso corporal médio de $34 \mathrm{~kg}$. Ao início do experimento, os animais foram pesados, vermifugados e alocados em quatro baias de piso ripado, recebendo água e sal mineral à vontade. 
Os animais foram designados aos tratamentos de forma totalmente aleatória, em função do peso vivo dos mesmos. Os tratamentos consistiram em diferentes relações volumoso:concentrado (V:C) da ração extrusada Lac24 ${ }^{\circledR}$ concentrado e Foragge ${ }^{\circledR}$ volumoso, conforme a Tabela 1 . A mesma era composta por dois tipos de pellets, sendo um do concentrado (Lac $24^{\circledR}$ ) e outro do volumoso $\left(\right.$ Foragge $^{\circledR}$ ). A ração veio misturada pelo fabricante nas diferentes proporções de $\mathrm{V}: \mathrm{C}$ em um único produto. A ração foi fornecida duas vezes ao dia as (8:00h e 16:00h), sendo que as sobras foram pesadas diariamente permitindo calcular o consumo pelos animais.

A cada quinze dias $(0,15,30,45,60,75$ e 90 dias do experimento) foram realizadas as seguintes medidas biométricas (Pinheiro e Jorge, 2010): circunferência do barril (CB), circunferência da porção ventral entre a décima e a décima primeira costela; circunferência torácica (CT), perímetro tomando-se como base o esterno (corpo) e a cernelha passando a fita métrica por trás da paleta; altura do posterior (AP), distância entre a tuberosidade sacral e a extremidade distal do membro posterior; altura do anterior (AA), distância entre a região da cernelha e a extremidade distal do membro anterior; largura do peito (LP), distância entre as faces laterais das articulações escápulo-umerais; comprimento corporal (CC), distância entre a articulação cérvico-torácica e a base da cauda e perímetro escrotal (PE) com o auxílio de fita métrica. 
Tabela 1 - Configuração dos tratamentos e composição bromatológica do Lac $24^{\circledR}$ e do Forrage $^{\circledR}$.

\begin{tabular}{ccc}
\hline Tratamentos & Concentrado - Lac $24^{\circledR}$ & Forrage $^{\circledR}$ - volumoso \\
\hline $70 \% \mathrm{C}: 30 \% \mathrm{~V}$ & $70 \%$ & $30 \%$ \\
$60 \% \mathrm{C}: 40 \% \mathrm{~V}$ & $60 \%$ & $40 \%$ \\
$50 \% \mathrm{C}: 50 \% \mathrm{~V}$ & $50 \%$ & $50 \%$ \\
$40 \% \mathrm{C}: 60 \% \mathrm{~V}$ & $40 \%$ & $60 \%$ \\
\hline Nutrientes & ${\text { Concentrado - LAC } 24^{\circledR *}}^{\circledR}$ & Forrage $^{\circledR *}(\%$ da MS) - volumoso \\
\hline PB (g/Kg) & 240 & 9,81 \\
NNP $(\mathrm{g} / \mathrm{Kg})$ & 35,0 & 30 \\
EE $(\mathrm{g} / \mathrm{Kg})$ & 200 & 1,50 \\
FDA $(\mathrm{g} / \mathrm{Kg})$ & 250 & 20,98 \\
FDN (g/Kg) & 143,3 & 32,27 \\
MM (g/Kg) & 100 & 8,27 \\
Monensina Sódica $(\mathrm{mg} / \mathrm{Kg})$ & 22,50 & - \\
\hline
\end{tabular}

${ }^{*}$ Dados fornecidos pelo fabricante Nutratta ${ }^{\circledR}$ (em \% da matéria seca). $\mathrm{PB}=$ proteína bruta; $\mathrm{NNP}=$ nitrogênio não proteico; $\mathrm{EE}=$ extrato etéreo; FDA = fibra em detergente ácido; FDN = fibra em detergente neutro; $\mathrm{MM}=$ matéria mineral; $\mathrm{MS}$ = matéria seca.

O escore de condição corporal (ECC) foi mensurado por meio da palpação da região dorso-lombar, baseando-se na sensibilidade da palpação à deposição de gordura e à musculatura nas vértebras. O ECC é uma avaliação subjetiva cujos escores variam de 1 a 5 , onde 1 representa uma condição corporal pobre, com pouca deposição de gordura e 5 representa uma condição corporal com deposição excessiva de gordura (EMBRAPA, 2008).

A pesagem dos animais também foi realizada quinzenalmente, medida em balança apropriada para a espécie, sempre antes da primeira oferta de alimento para a determinação do ganho de peso. A mensuração do ganho médio diário (GMD) foi calculada tomando-se o peso inicial e final de cada período experimental, e dividido pelo tempo transcorrido.

Avaliou-se também o comportamento ingestivo, onde os animais foram observados por pessoas treinadas, localizadas de maneira que não provocasse estresse aos animais. Tais observações foram realizadas a cada 5 minutos, durante 24 horas, no início e no final do período experimental, seguindo a metodologia proposta por Fischer et al., (1998). A observação dos animais foi feita para identificação de ócio, ruminação e ingestão. A observação noturna dos animais foi realizada com o auxílio de iluminação artificial de lâmpadas frias, de modo a não provocar alterações no 
comportamento ingestivo dos animais. Os cálculos das atividades foram feitos em minutos por dia, considerando que, nos cinco minutos subsequentes a cada observação, o animal permaneceu na mesma atividade. Já o tempo total de mastigação foi determinado somando-se os tempos de alimentação e ruminação como citado por Bal e Büyükünal Bal (2009).

$\mathrm{O}$ delineamento experimental utilizado foi inteiramente ao acaso em arranjo fatorial (sexo $\mathrm{x}$ níveis) com parcelas subdivididas. Nas parcelas ficaram as avaliações em função do tempo. As variáveis foram comparadas por equação de regressão a 5\% de probabilidade. As médias referentes a sexo foram comparadas pelo teste SNK a 5\% de probabilidade. Cada baia recebeu três fêmeas e dois machos (devidamente equilibrados em peso para não afetar o estudo). Todas as variáveis apresentaram normalidade e homogeinicidade. A análise de escore corporal foi feita por estatística não paramétrica.

\section{Resultados e discussão}

Não houve efeito significativo $(\mathrm{P}>0,05)$ nas diferentes relações $\mathrm{V}: \mathrm{C}$ sobre o escore de condição corporal (ECC) e nem sobre nenhum dos parâmetros biométricos mensurados no experimento (Tabela 2). Em relação ao efeito do sexo, não houve diferença estatística para as medidas biométricas, porém observou-se efeito para o $\operatorname{ECC}(\mathrm{P}<0,05)$, destacando-se superioridade para as fêmeas. Isto se deve ao fato das fêmeas serem mais precoces que os machos e, portanto, tendem a depositar uma maior quantidade de gordura em relação aos machos de mesma idade ou peso corporal (PELLEGRINI et al., 2012). Além disso, o comportamento sexual dos machos não castrados também pode ter influenciado a deposição de gordura, visto que muitas vezes deixam de se alimentar devido a disputa pelo território e pelas fêmeas. Devido a isso, o ECC é totalmente influenciado pela deposição tecidual do animal. 
Tabela 2 - Efeito da utilização da ração extrusada em diferentes relações V:C sobre medidas biométricas $(\mathrm{cm})$ e escore de condição corporal (ECC) em ovinos de classes sexuais distintas e em diferentes períodos (dias).

\begin{tabular}{llllllll}
\hline Tratamento & ECC $^{*}$ & CB & CT & AP & AA & LP & CC \\
\hline $70 \% \mathrm{C}: 30 \% \mathrm{~V}$ & 3,08 & 77,71 & 68,92 & 57,92 & 57,45 & 18,69 & 57,50 \\
$60 \% \mathrm{C}: 40 \% \mathrm{~V}$ & 3,08 & 80,85 & 71,26 & 56,54 & 56,97 & 19,04 & 57,54 \\
$50 \% \mathrm{C}: 50 \% \mathrm{~V}$ & 3,21 & 83,25 & 72,51 & 57,71 & 57,14 & 19,88 & 58,20 \\
$40 \% \mathrm{C}: 60 \% \mathrm{~V}$ & 3,21 & 86,25 & 73,08 & 59,88 & 59,34 & 20,77 & 59,42 \\
\hline Sexo & $\mathrm{ECC}^{*}$ & $\mathrm{CB}$ & $\mathrm{CT}$ & $\mathrm{AP}$ & $\mathrm{AA}$ & $\mathrm{LP}$ & $\mathrm{CC}$ \\
\hline Macho & $2,94 \mathrm{~B}$ & 80,05 & 68,62 & 56,97 & 56,51 & 18,94 & 57,50 \\
Fêmea & $3,30 \mathrm{~A}$ & 83,20 & 73,57 & 58,76 & 58,65 & 20,02 & 58,61 \\
\hline Período & $\mathrm{ECC}^{*}$ & $\mathrm{CB}^{1}$ & $\mathrm{CT}^{2}$ & $\mathrm{AP}^{3}$ & $\mathrm{AA}^{4}$ & $\mathrm{LP}^{5}$ & $\mathrm{CC}^{6}$ \\
\hline 0 & $2,84 \mathrm{~A}$ & 70,31 & 61,59 & 54,22 & 54,22 & 15,68 & 51,86 \\
15 & $3,02 \mathrm{AB}$ & 74,68 & 65,13 & 55,59 & 55,50 & 16,72 & 53,77 \\
30 & $3,25 \mathrm{ABC}$ & 81,68 & 69,27 & 55,27 & 56,09 & 19,00 & 57,09 \\
45 & $3,20 \mathrm{BCD}$ & 81,72 & 73,09 & 58,09 & 57,59 & 19,59 & 59,22 \\
60 & $3,00 \mathrm{DC}$ & 85,13 & 74,72 & 59,86 & 59,18 & 21,13 & 59,81 \\
75 & $3,15 \mathrm{D}$ & 88,31 & 76,04 & 60,68 & 60,04 & 21,54 & 62,18 \\
90 & $3,52 \mathrm{E}$ & 90,54 & 79,40 & 61,90 & 61,13 & 23,03 & 62,81 \\
\hline MG & 3,14 & 81,77 & 71,32 & 57,94 & 57,68 & 19,53 & 58,11 \\
CV & - & 8,22 & 9,26 & 7,57 & 7,17 & 10,48 & 6,96 \\
\hline
\end{tabular}

${ }^{*}$ Estatística não paramétrica. ${ }^{1} \mathrm{Y}: 70,607143+0,327381 \mathrm{X}-0,001219 \mathrm{X}^{2}, \mathrm{R}^{2}=97,48 \% ;{ }^{2} \mathrm{Y}: 61,522727+0,284416 \mathrm{X}-$ $0,001025 \mathrm{X}^{2}, \mathrm{R}^{2}=99,01 \% ;{ }^{3} \mathrm{Y}: 53,896104+0,090043 \mathrm{X}, \mathrm{R}^{2}=95,63 \% ;{ }^{4} \mathrm{Y}: 54,155844+0,078355 \mathrm{X}, \mathrm{R}^{2}=99,17 \%$; ${ }^{5} \mathrm{Y}: 15,598485+0,105087 \mathrm{X}-0,000272 \mathrm{X}^{2}, \mathrm{R}^{2}=98,38 \%,{ }^{6} \mathrm{Y}: 51,648268+0,192532 \mathrm{X}-0,000753 \mathrm{X}^{2}, \mathrm{R}^{2}=98,75 \%$. Letras distintas na coluna diferem entre si pelo teste de SNK a $5 \%$.

O ECC foi influenciado pelos períodos de avaliação, fato decorrente do crescimento e desenvolvimento dos animais ao longo do experimento (Tabela 2). O período 0 diferenciou-se dos outros do $45^{\circ}$ dia de avaliação em diante. No dia 90, a média de ECC foi maior quando comparados aos dias anteriores, fato lógico devido à idade dos animais à época de avaliação, que era de aproximadamente 4 meses, fase esta que se caracteriza pelo pleno crescimento do animal. Esta curva observada no ECC esta relacionada também ao tempo de deposição dos diferentes tipos de tecido, uma vez que primeiro se dá o crescimento ósseo, seguido pelo crescimento muscular, e por último deposição de gordura intramuscular (ALVES et al., (2015). Além disso, os menores valores de ECC dos machos podem ter contribuído para a queda dos valores médios gerais no período inicial da puberdade, uma vez que nesta fase eles são muito ativos e muitas vezes deixam de se alimentar atrapalhando até outros animais na alimentação em busca da cópula. 
Constatou-se efeito dos períodos sobre as medidas biométricas $(\mathrm{P}>0,05)$, essas apresentaram equações lineares e quadráticas, indicando aumento progressivo das medidas ao longo do período experimental, fato este também em função dos argumentos discutidos acima.

Houve interação $(\mathrm{P}<0,05)$ de todos os tratamentos testados com os diferentes períodos de avaliação para perímetro escrotal (Tabela 3), apresentando equações quadráticas e lineares. Observa-se que os testículos cresceram de maneira normal apresentando estabilização no desenvolvimento a partir de 75 dias de experimento. Esse comportamento pode estar relacionado ao início da fase de puberdade, caracterizada por possível diminuição do consumo de alimento pelos machos. Segundo Mancio et al. (2005) em organismos em crescimento, um inadequado suprimento de nutrientes reduz a divisão celular dos tecidos e dos órgãos, afetando assim, o perímetro escrotal. Neste sentido, o tratamento 60C:40V se mostrou mais eficaz no que se refere à manutenção desta característica pois, apresentou maior perímetro escrotal aos 90 dias de experimento.

Tabela 3 - Interação entre os tratamentos e os períodos (dias) para perímetro escrotal (cm).

\begin{tabular}{cccccccc}
\hline Tratamento & 0 & 15 & 30 & 45 & 60 & 75 & 90 \\
\hline $70 \% \mathrm{C}: 30 \% \mathrm{~V}^{1}$ & 11,66 & 15,33 & 17,66 & 24,00 & 26,66 & 29,66 & 28,66 \\
$60 \% \mathrm{C}: 40 \% \mathrm{~V}^{2}$ & 13,00 & 16,33 & 19,33 & 23,00 & 25,66 & 28,66 & 30,33 \\
$50 \% \mathrm{C}: 50 \% \mathrm{~V}^{3}$ & 13,50 & 16,00 & 22,00 & 24,50 & 26,00 & 25,50 & 24,00 \\
$40 \% \mathrm{C}: 60 \% \mathrm{~V}^{4}$ & 14,50 & 18,00 & 21,00 & 24,00 & 26,50 & 28,00 & 29,00 \\
\hline${ }^{1} \mathrm{Y}: 11,841270+0,30000 \mathrm{X}-0,001093 \mathrm{X}^{2}, \mathrm{R}^{2}=95,61 \% ;{ }^{2} \mathrm{Y}: 12,821429+0,207143 \mathrm{X}, \mathrm{R}^{2}=98,59 \% ;$ \\
${ }^{3} \mathrm{Y}: 12,559524+0,389286 \mathrm{X}-0,002884 \mathrm{X}^{2}, \mathrm{R}^{2}=96,97 \% ;{ }^{4} \mathrm{Y}: 15,607143+0,164286 \mathrm{X}, \mathrm{R}^{2}=96,89 \%$. \\
$\mathrm{MG}=22,12 ; \mathrm{CV}=11,2$
\end{tabular}

Não se observou efeito das diferentes relações V:C e nem do sexo sobre o peso dos animais em experimentação (Tabela 4). No entanto, como esperado, o peso dos animais foi influenciado pelo período, pois os animais se encontravam em plena fase de crescimento. Comparando os resultados, Cunha et al. (2008) utilizando cordeiros alimentados com caroço de algodão, com idade de aproximadamente 6 meses, obtiveram média de 33,5 kg de peso médio corporal. Na Tabela 4 observa-se que houve ganho de peso 
eficiente de forma que os animais chegaram ao final do experimento com peso adequado para o abate (em torno de $40 \mathrm{~kg}$ ) respondendo favoravelmente a inclusão da ração extrusada.

Tabela 4 - Efeito do tratamento, sexo e período sobre o peso (kg) dos animais.

\begin{tabular}{cc}
\hline Tratamento & Peso (kg) \\
\hline $70 \% \mathrm{C}: 30 \% \mathrm{~V}$ & 31,07 \\
$60 \% \mathrm{C}: 40 \% \mathrm{~V}$ & 32,10 \\
$50 \% \mathrm{C}: 50 \% \mathrm{~V}$ & 34,20 \\
$40 \% \mathrm{C}: 60 \% \mathrm{~V}$ & 36,50 \\
\hline Sexo & Peso (kg) \\
\hline Macho & 34,78 \\
Fêmea & 31,52 \\
\hline Período (dias) & Peso (kg) \\
\hline 0 & 21,30 \\
15 & 25,66 \\
30 & 30,16 \\
45 & 33,81 \\
60 & 37,11 \\
75 & 40,98 \\
90 & 44,06 \\
\hline $\mathrm{MG}$ & 33,30 \\
$\mathrm{CV}$ & 14,79 \\
\hline${ }^{1} \mathrm{Y}: 21,343723+0,301223 \mathrm{X}+0,000546 \mathrm{X}^{2} ; \mathrm{R}^{2}=99,95 \%$ &
\end{tabular}

Não houve efeito $(\mathrm{P}>0,05)$ das diferentes relações de $\mathrm{V}: \mathrm{C}$ da ração extrusada sobre ganho médio diário em nenhum dos períodos avaliados (Tabela 5). Observou-se diferenças significativas das distintas classes sexuais sobre o ganho médio diário (GMD) nos períodos 15-30, 0-30 e 75-90 dias, sendo que nestes períodos os machos apresentaram superioridade no ganho em comparação com as fêmeas (Tabela 5). Isto se deve ao fato de que as fêmeas, em período fisiológico de puberdade, decrescem o ritmo de ganho de peso para depositarem mais gordura, em contrapartida os machos apresentam maior proporção de osso e músculo devido à ação do hormônio testosterona em que é precursor do desenvolvimento muscular, atuando também sobre a composição da carcaça de cordeiros. Este resultado está relacionado com a superioridade do ECC observado nas fêmeas (Tabela 2). Corroborando a afirmação, Rodríguez et al. (2008) trabalhando com cordeiros Assaf, também encontrou superioridade em ganho médio diário pelos machos em relação às fêmeas. 
Tabela 5 - Efeito do sexo e das diferentes relações V:C nos períodos de avaliação sobre o ganho médio diário (GMD em gramas).

\begin{tabular}{lllllll}
\hline \multirow{2}{*}{ Tratamento } & \multicolumn{6}{l}{ Intervalo entre períodos (dias) } \\
\cline { 2 - 7 } & $0-15$ & $15-30$ & $0-30$ & $30-45$ & $45-60$ & $75-90$ \\
\hline $70 \% \mathrm{C}: 30 \% \mathrm{~V}$ & 238,88 & 288,89 & 263,89 & 281,11 & 183,33 & 282,05 \\
$60 \% \mathrm{C}: 40 \% \mathrm{~V}$ & 303,33 & 297,77 & 300,55 & 217,77 & 243,33 & 235,89 \\
$50 \% \mathrm{C}: 50 \% \mathrm{~V}$ & 294,66 & 312,00 & 303,33 & 253,33 & 169,33 & 203,96 \\
$40 \% \mathrm{C}: 60 \% \mathrm{~V}$ & 334,66 & 305,33 & 319,99 & 218,66 & 286,66 & 247,69 \\
\hline Macho & 316,66 & $341,33 \mathrm{~A}$ & $329,00 \mathrm{~A}$ & 264,00 & 249,33 & $291,98 \mathrm{~A}$ \\
Fêmea & 269,44 & $266,33 \mathrm{~B}$ & $267,66 \mathrm{~B}$ & 226,11 & 195,55 & $203,84 \mathrm{~B}$ \\
\hline MG & 290,90 & 300,30 & 295,60 & 295,60 & 220,00 & 243,90 \\
CV & 28,34 & 15,31 & 13,79 & 13,79 & 34,32 & 33,20 \\
\hline
\end{tabular}

Letras distintas na mesma coluna diferem entre si pelo teste de Tukey $(\mathrm{P}<0,05)$.

$\mathrm{Na}$ tabela 6 observa-se a interação entre classe sexual e o período de avaliação. Verifica-se que os machos apresentaram comportamento do ganho médio diário superior as fêmeas em diversos períodos. Na relação 60:40 houve crescimento linear para machos e fêmeas em função dos períodos analisados. O GPD do presente neste estudo é superior aos encontrados na literatura quando utilizam-se alimentos convencionais (MURTA et al. 2009; BARROS et al., 1994). A superioridade dos machos em relação às fêmeas quanto ao ganho de peso também foi vista por Zidane, Niar e Ababou (2015) trabalhando com cordeiros de 0 a 90 dias.

Tabela 6 - Relação dos tratamentos e efeito do sexo em função dos períodos para ganho médio diário (GMD em gramas).

\begin{tabular}{|c|c|c|c|c|c|c|}
\hline \multirow{2}{*}{$\begin{array}{l}\text { Tratamento } \\
(\% \mathrm{C}: \mathrm{V})\end{array}$} & \multirow{2}{*}{ Sexo } & \multicolumn{5}{|c|}{ Período (dias) } \\
\hline & & $0-45$ & $0-60$ & $60-75$ & $0-75$ & $0-90$ \\
\hline \multirow{2}{*}{$70: 30$} & Macho & 290,37 & 266,11 & $340,00 \mathrm{~A}$ & $280,89 \mathrm{~A}$ & 285,82 \\
\hline & Fêmea & 248,89 & 230,00 & $188,89 \mathrm{~B}$ & 221,78 B & 231,80 \\
\hline \multirow{2}{*}{$60: 40$} & Macho $^{1}$ & $334,81 \mathrm{~A}$ & $329,44 \mathrm{~A}$ & 275,55 & $318,66 \mathrm{~A}$ & $317,62 \mathrm{~A}$ \\
\hline & Fêmea ${ }^{2}$ & $211,11 \mathrm{~B}$ & $201,66 \mathrm{~B}$ & 220,23 & $194,66 \mathrm{~B}$ & $195,40 \mathrm{~B}$ \\
\hline \multirow{2}{*}{$50: 50$} & Macho & 283,33 & 250,83 & 262,50 & 230,66 & 226,43 \\
\hline & Fêmea & 288,89 & 261,66 & 277,77 & 264,89 & 252,87 \\
\hline \multirow{2}{*}{$40: 60$} & Macho & 315,55 & 320,00 & 300,00 & 316,00 & 321,26 \\
\hline & Fêmea & 266,66 & 263,89 & 342,22 & 279,89 & 270,11 \\
\hline \multirow{2}{*}{$\begin{array}{l}\text { MG } \\
\text { CV }\end{array}$} & & 278,18 & 263,11 & 275,40 & 262,48 & 285,82 \\
\hline & & 11,36 & 12,86 & $19,08^{1}$ & $11,81^{2}$ & 13,73 \\
\hline
\end{tabular}

${ }^{1} \mathrm{Y}: 240,3830+5,175433 \mathrm{X}, \mathrm{R}^{2}=97,86 \% ;{ }^{2} \mathrm{Y}: 130,6280+2,435433 \mathrm{X}, \mathrm{R}^{2}=64,88 \%$. Letras distintas na mesma coluna, dentro de cada tratamento, diferem significativamente pelo teste de Tukey $(\mathrm{P}<0,05)$. 
Não foram observados efeitos dos tratamentos para variável ócio. Houve interação entre sexo e período para a variável ócio (Tabela 7) no qual demonstra que o tempo em ócio foi menor no início do experimento. Esse comportamento ocorreu pelo fato dos animais fazerem maior consumo ao início do experimento. A ração extrusada é facilmente consumida e palatável aos animais, o que facilita e estimula o consumo pelos mesmos. O maior valor de ócio tanto para machos quanto para fêmeas ao final do período deve-se a estabilização do consumo que ocorreu na fase final do experimento.

Tabela 7 - Médias do tempo gasto com as atividades ingestivas (ócio e mastigação em minutos/dia) em função da interação entre classe sexual e período.

\begin{tabular}{|c|c|c|c|c|}
\hline $\begin{array}{l}\text { Tratamento } \\
(\% \mathrm{C}: \mathrm{V})^{*}\end{array}$ & Ócio & \multicolumn{3}{|c|}{ Interação entre sexo e período para ócio } \\
\hline $70: 30$ & 1035,41 & \multirow{4}{*}{$\begin{array}{l}\text { Período } \\
\text { Início } \\
\text { Final }\end{array}$} & Macho & Fêmea \\
\hline $60: 40$ & 1079,16 & & 1010,00B & $1032,08 \mathrm{~B}$ \\
\hline $50: 50$ & 1082,00 & & $1106,50 \mathrm{~A}$ & $1090,50 \mathrm{~A}$ \\
\hline $40: 60$ & 1044,00 & & $\mathrm{CV}=8,82$ & $\mathrm{MG}=1060,00$ \\
\hline \multicolumn{5}{|c|}{ Interação entre sexo e período para mastigação } \\
\hline \multicolumn{2}{|c|}{ Sexo } & \multicolumn{2}{|c|}{ Início } & Final \\
\hline \multicolumn{2}{|l|}{ Macho } & \multicolumn{2}{|r|}{$430,00 \mathrm{a}$} & $333,50 \mathrm{~b}$ \\
\hline \multicolumn{2}{|l|}{ Fêmea } & \multicolumn{2}{|r|}{$407,91 \mathrm{a}$} & $349,16 \mathrm{~b}$ \\
\hline
\end{tabular}

*Ração extrusada em diferentes relações volumoso concentrado; MG, média geral; CV, coeficiente de variação. Letras maiúsculas nas colunas e minúsculas nas linhas apresentam diferença estatística $(\mathrm{P}<0,05)$ pelo teste Tukey.

Machos e fêmeas apresentaram maior valor de mastigação ao início do experimento e redução ao final. Essa informação corrobora com a discussão feita acima para a variável ócio.

$\mathrm{Na}$ interação entre tratamento e período para ingestão (Tabea 8) podese observar que esta demonstra que no início do experimento os animais passaram mais tempo realizando ingestão, com efeito significativo para as relações 40:60 e 50:50. A média do tempo gasto com alimentação foi menor (aproximadamente $3,15 \mathrm{~h} /$ dia) em relação ao encontrado na literatura, em que o tempo de alimentação, em pastejo, é de aproximadamente 8 horas (REIS e Da SILVA, 2006). No entanto em condições de confinamento, com animais recebendo ração extrusada de fácil seleção e apreensão, o tempo gasto com alimentação tende a ser menor, o que permite aos animais maior velocidade de ingestão da ração e menor tempo para atingir o ponto de 
saciedade. Segundo Carvalho e Moraes (2005), alta disponibilidade de alimentos de qualidade induz ao maior número de refeições em menor tempo, fazendo com que a velocidade de ingestão aumente com consequente enchimento do rúmen. O tempo de ingestão de alimentos são indicadores de qualidade do alimento, pois esse tempo irá variar de acordo com o tamanho de partícula, qualidade e nível de facilidade para apreensão dos alimentos. Assim, infere-se que a ração extrusada no experimento era de boa qualidade e de fácil consumo pelos animais, como já discutido anteriormente.

Tabela 8 - Médias do tempo gasto com as atividades ingestivas (ingestão, ruminação e mastigação em minutos/dia) em função da interação entre dos tratamentos e período.

\begin{tabular}{|c|c|c|c|c|c|c|}
\hline \multicolumn{7}{|c|}{ Interação entre tratamento e período para ingestão } \\
\hline Sexo & Ingestão & Período & $70: 30$ & $60: 40$ & $50: 50$ & $40: 60$ \\
\hline Macho & 197,25 & Início & 200,83 & 200,83 & $219,00 \mathrm{~A}$ & $200,00 \mathrm{~A}$ \\
\hline \multirow[t]{2}{*}{ Fêmea } & 181,87 & Final & 209,16 & 183,33 & $140,00 \mathrm{~B}$ & $150,00 \mathrm{~B}$ \\
\hline & & & $\mathrm{CV}=10,40$ & \multicolumn{3}{|c|}{$\mathrm{MG}=188,86$} \\
\hline \multicolumn{7}{|c|}{ Interação entre tratamento e período para ruminação } \\
\hline Sexo & Ruminação & Período & $70: 30$ & $60: 40$ & $50: 50$ & $40: 60$ \\
\hline Macho & 184,50 & Início & $275,83 \mathrm{~A}$ & 167,50 & 158,00 & 248,00 \\
\hline \multirow[t]{2}{*}{ Fêmea } & 196,66 & Final & $123,33 \mathrm{~B}$ & 170,00 & 190,00 & 193,00 \\
\hline & & & $\mathrm{CV}=28,08$ & \multicolumn{3}{|c|}{$\mathrm{MG}=191,13$} \\
\hline \multicolumn{7}{|c|}{ Interação entre tratamento e período para mastigação } \\
\hline Mastigação & & & $70: 30$ & $60: 40$ & $50: 50$ & $40: 60$ \\
\hline $\mathrm{CV}=6,25$ & & Início & $476,66 \mathrm{a}$ & 368,33 & $377,00 \mathrm{a}$ & $448,00 \mathrm{a}$ \\
\hline $\mathrm{MG}=380$ & & Final & $332,50 \mathrm{~b}$ & 353,33 & $339,00 b$ & $343,00 \mathrm{~b}$ \\
\hline
\end{tabular}

MG, média geral; CV, coeficiente de variação. Letras maiúsculas nas colunas e minúsculas nas linhas apresentam diferença estatística $(\mathrm{P}<0,05)$ pelo teste Tukey.

Observou-se diferença estatística $(\mathrm{P}<0,05)$ na interação entre tratamento e período para ruminação indicando que houve maior tempo de atividade de ruminação, associado ao maior tempo de ingestão no inicio da fase experimental. A média geral, em horas, foi de 3,18 de ruminação o que é considerado baixo. Van Soest (1994) estabeleceu que a atividade de ruminação em animais adultos deve ocupar em torno de 8 horas por dia, podendo apresentar variações entre 4 e 9 horas. De acordo com Furlan, Macari e Faria Filho (2006), esta variação é dependente da estrutura do alimento, incluindo o teor de fibra, tamanho de partícula e também do número de refeições e quantidade de alimento ingerido. Ainda segundo estes autores, o tempo gasto com ruminação é em média de 7 horas. O processo de 
extrusão da ração testada proporciona tamanho de partícula de $2 \mathrm{~mm}$, o que possivelmente levou à redução do tempo de ruminação, pois desta forma houve elevação da taxa de passagem do alimento no trato gastrointestinal com consequente redução da ruminação. O processo de ruminação auxilia na manutenção do pH ruminal através da produção de saliva. Sendo assim, queda brusca no tempo de ruminação pode provocar queda do $\mathrm{pH}$ ruminal, causando possíveis problemas, como a acidose. Entretanto, nenhum desses problemas foi verificado no experimento.

A mastigação total teve interação para tratamento e período, onde o tempo gasto em mastigação foi maior no início do período, devido o maior consumo no período inicial em relação ao período final tanto para os machos quanto para as fêmeas. Isso se deve também à influência do pequeno tamanho de partícula da fibra contida na ração extrusada.

\section{Conclusões}

As diferentes relações concentrado: volumoso testadas promoveram desempenho igual aos animais, sem afetar de maneira deletéria o comportamento ingestivo dos animais. A extrusão do volumoso e concentrado é uma ferramenta interessante para maximização do desempenho de ovinos 


\title{
Evaluation of performance and ingestive behavior of sheep fed extruded ration in different forage:concentrate ratios
}

\begin{abstract}
The objective was to evaluate the use of extruded ration in different forage:concentrate (R:C) ratios and its effect on the ingestive behavior, biometric measurements and weight gain of sheep of different sex classes. The experiment was carried out at the Experimental Farm Capim Branco, belonging to the Federal University of Uberlândia, from July to September 2015, 15 days for the adaptation of animals and 90 days for data collection. Twenty crossbred $1 / 2$ Dorper and $1 / 2$ Santa Inês sheep were used, being eight males and twelve females, of approximately four months old and body weight of $34 \mathrm{~kg}$. The treatments consisted of different V: C extrudate ratios: $70 \mathrm{C}: 30 \mathrm{~V}, 60 \mathrm{C}: 40 \mathrm{~V}, 50 \mathrm{C}: 50 \mathrm{~V}, 40 \mathrm{C}: 60 \mathrm{~V}$. Biometric measurements and weight gain were measured every 15 days using appropriate tape measure, ruler and balance. Ingestive behavior was performed at the beginning and end of the experimental period. The experimental design was completely randomized in factorial arrangement (sex x levels) with subdivided plots. The variables were compared by regression equation at $5 \%$ probability. The means relative to sex were compared by the SNK test at $5 \%$ probability. There was no effect of the different V:C relationships on the body condition score (BCS) and on the biometric parameters. The BCS was influenced by the evaluation periods and the sex of the animals. Significant differences were observed between the different sex classes over the average daily gain, with male superiority. There was interaction between sex and period for leisure, treatment and period for rumination and interaction between treatment and feeding period, the latter being higher in the initial periods. The extruded ration causes changes in the body condition of the sheep, positively affecting the weight gain and altering the ingestive behavior.
\end{abstract}

Key words: Biometry, Extrusion, Weight gain, Ovis Aries

\section{Referências}

ALVES, G.C.A.; OSÓRIO, J.C.S.; OSÓRIO, M.T.M.; FERNANDES, A.R.M.; RIBEIRO, E.L.A.; DA CUNHA, C.M.; ALMEIDA, H.R.; FUZIKAWA, I.H.S. Avaliação da composição regional e tecidual da carcaça ovina. Publicações em Medicina Veterinária e Zootecnia, Maringá, v.9, n.1, p. 6-19, jan., 2015. https://doi.org/10.22256/pubvet.v9n1.6-19 
AMARAL, C. M. C.; PELICANO, E. R. L.; YAÑEZ, E. A.; SOUZA, H. B. A.; MACHADO, M. R. F.; SUGOHARA, A.; RESENDE, K. T. Características de carcaça e qualidade de carne de cabritos Saanen alimentados com ração completa farelada, peletizada e extrusada. Ciência Rural, Santa Maria, v. 37, n. 2, p. 550556, mar/abr. 2007. https://doi.org/10.1590/S0103-84782007000200040

BAL, M. A.; BÜYÜKÜNAL BAL, E. B. Interaction between particle sizes of alfalfa hay and corn grain on milk yield, milk composition, chewing activity, and ruminal $\mathrm{pH}$ of dairy cows. Turkish Journal of Veterinary and Animal Sciences, Kahramanmaraş, Turquia, v. 34, n. 1, p. 83-89, jan. 2009.

BARROS, N.N.; FIGUEIREDO, E.A.P.; FERNANDES, F.D.; BARBIERI, M.E. Ganho de peso e conversão alimentar de cordeiros cruzas do estado do Ceará. Pesq. Agrop. Brás., v. 29, n. 8, p. 1313-1317, 1994.

CARVAlHO, P. C. F.; MORAES, A. Comportamento ingestivo de Ruminantes: bases para o manejo sustentável do pasto. In: MANEJO SUSTENTÁVEL EM PASTAGEM, 1., 2005, Maringá. Anais... Maringá: UEM, 2005, p. 1-20.

COELHO DA SILVA, J. F. Mecanismos reguladores de consumo. In: BERCHIELLI, T. T.; PIRES A. V.; OLIVEIRA, S. G. (Eds.) Nutrição de Ruminantes. Jaboticabal: Funep, 2006. p. 57-78.

CUnHA, M. G. C.; CARVAlhO, F. F. R.; GOnZAGA NETO, S.; CEZAR, M. F. Características quantitativas de carcaça de ovinos Santa Inês confinados alimentados com rações contendo diferentes níveis de caroço de algodão integral. Revista Brasileira de Zootecnia, v.37, n.6, p.1112-1120, 2008. https://doi.org/10.1590/S1516-35982008000600023

CYRILLO, J. N. S. G.; NARDON, R. R.; MERCADANTE, M. E. Z.; BONILHA, S. F. M.; ARNANDES, R. H. B. Relações entre medidas biométricas, características de carcaça e cortes cárneos comerciais em bovinos zebu e caracu. Boletim de Indústria Animal, Nova Odessa, v. 69, n. 1, p. 71-77, jan./jun. 2012. 
EMBRAPA. Circular técnica 57: Escore da condição corporal e sua aplicação no manejo reprodutivo de ruminantes. São Carlos, 2008. 16 p.

FURLAN, R. L.; MACARI, M.; FARIA FILHO, D. E. Anatomia e fisiologia do trato gastrintestinal. In: BERCHIELLI, T. T.; PIRES A. V.; OLIVEIRA, S. G. (Eds.) Nutrição de Ruminantes. Jaboticabal: Funep, 2006. p. 1-27.

KANITZ, F.; MORGÃO, G.; KLAESNER, A. L.; PORT, C.; SILVEIRA, J.; DAMIANI, J.; BORGES, L. F. K. Avaliação da condição corporal em ovinos e a correlação com produção de carne. In: SEMINÁRIO INTERINSTITUCIONAL DE ENSINO, PESQUISA E EXTENSÃO, 19., 2014, Cruz Alta. Anais... Cruz Alta: UNICRUZ, 2014.

LIMA JÚNIOR, D. M.; BRAGA, A. P.; RANGEL, A. H. N.; BRAGA, Z. C. A. C.; BARRETO, H. F. M.; MACIEL, M. V. Farelo de algodão (Gossipum spp.) extrusado na dieta de ruminantes: consumo e digestibilidade. Acta Veterinária Brasílica, v. 5, n. 1 , p. $68-75,2011$.

MANCIO, A.B.; SANTIAGO, L.L.; GOES, R.H.T.B.; MARTINS, L.F.; CECON, P.R. Perímetro scrotal e idade à puberdade em ovinos Merino Australiano submetidos a diferentes regimes alimentares. Acta Sci. Anim. Sci., v. 27, n. 4, p. 449-457, 2005. https://doi.org/10.4025/actascianimsci.v27i4.1179

MURTA, R.M.; CHAVES, M.A.; SILVA, F.V.; BUTERI, C.B.; FERNANDES, O.W.B.; SANTOS, L.X. Ganho de peso e características de carcaça de ovinos confinados alimentados com bagaço de cana hidrolisado com óxido de cálcio. Ciência Animal Brasileira, v. 10, n. 2, p. 438-455, 2009.

PELLEGRINI, L. G.; PELLEGRIN, A. C. R. S.; MONTEIRO, A. L. G.; NEUMANN, M.; PELLEGRINI, L. G. Efeito do sexo no desempenho de cordeiros desmamados terminados em pasto de azevém. In: SIMPÓSIO PARANAENSE DE OVINOCULTURA, 15., 2012, Pato Branco. Anais... Pato Branco: UTFPR, 2012. 1 CD-Rom. 
PINHEIRO, R. S. B.; JORGE, A. M. Medidas biométricas obtidas in vivo e na carcaça de ovelhas de descarte em diferentes estágios fisiológicos. Revista Brasileira de Zootecnia, v. 39, n. 2, p. 440-445, 2010. https://doi.org/10.1590/S1516$\underline{35982010000200030}$

REIS, R. A.; DA SILVA, S. C. Consumo de forragens. In: BERCHIELLI, T. T.; PIRES A. V.; OLIVEIRA, S. G. (Eds.) Nutrição de Ruminantes. Jaboticabal: Funep, 2006. p. 83-114.

RODRÍGUEZ, A. B.; BODAS, R.; PRIETO, N.; LANDA, R.; MANTECÓN, A. R.; GIRÁLDEZ, F. J. Effect of sex and feeding system on feed intake, growth, and meat and carcass characteristics of fattening Assaf lambs. Livestock Science, v. 116, n. 13, p. 118-125, 2008. https://doi.org/10.1016/j.livsci.2007.09.016

VAN SOEST, P.J. Nutritional Ecology of the Ruminant, $2^{\circ}$ ed. Cornell University Press, Ithaca, NY, 1994. 476p.

WERNERSBACH FILHO, H. L. Rações farelada, peletizada e extrusada na alimentação e produção de vacas leiteiras. 2003. 56 p. Dissertação (Mestrado em Zootecnia) - Universidade Federal de Viçosa, Viçosa, MG, 2003.

ZIDANE, A.; NIAR, A.; ABABOU, A.; Effect of some factors on lambs growth performance of the Algerian Ouled Djellal breed. Livestock Research for Rural Development, v. 27, n. 7, 2015. 